\title{
Development of shellfish allergy after exposure to dual immune checkpoint blockade
}

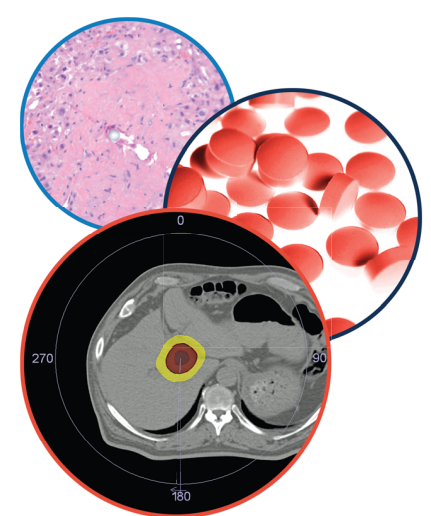

Hepatic Oncology

Zachary J Brown*,1, Bernd Heinrich ${ }^{1}$ \& Tim F Greten ${ }^{1}$

${ }^{1}$ Gastrointestinal Malignancy Section, Thoracic \& Gastrointestinal Oncology Branch, Center for Cancer Research, National Cancer Institute, National Institutes of Health, Bethesda, MD 20892, USA

*Author for correspondence: Tel.: +301 496 0928; zachary.brown@nih.gov

Hepatocellular carcinoma (HCC) is a major health problem worldwide with limited systemic therapies available. Immunotherapy is a fast-moving field that is quickly evolving as a treatment for HCC with three recent clinical trials published treating $\mathrm{HCC}$ with immune checkpoint inhibitors with promising results. Checkpoint inhibition may lead to a unique adverse event profile with the potential to cause immunerelated adverse events by unbalancing the immune system. Here, we report a case of a 61-year-old male with advanced HCC who developed a shellfish allergy after completing three cycles of combination of tremelimumab and durvalumab therapy.

First draft submitted: 26 September 2017; Accepted for publication: 17 January 2018; Published online: 9 February 2018

Keywords: allergy • checkpoint inhibitors • durvalumab • hepatocellular carcinoma • immunotherapy • tremelimumab

Hepatocellular carcinoma (HCC) is a major health problem worldwide being the second leading cause of cancerrelated deaths with the highest increasing incidence of all cancer sites [1]. The standard of care for HCC not amenable to local therapies such as resection, ablation or liver transplantation is sorafenib as well as regorafenib showing a slight survival advantage over placebo $[2,3]$. Thus far, three clinical trials that have been published treating HCC with immune checkpoint inhibitors with promising results and acceptable safety profiles. Unlike traditional chemotherapy that has direct cytotoxic effects on cancer cells, immunotherapy acts indirectly through modulating the patient's immune system to promote tumor recognition and an antitumor response [4]. The immune system is designed with an elegant system of checks and balances to prevent widespread immune activation and subsequent autoimmune disease. Immunotherapy may produce a unique adverse event profile with the potential to cause immune-related adverse events (irAEs) by unbalancing the natural state of equilibrium where one may develop autoimmune manifestations [5]. Therefore, autoimmune toxicity must be considered when using any form of immunotherapy, including checkpoint inhibitors, which may lead to irAEs.

Currently at the National Institutes of Health, a clinical trial is ongoing treating patients with HCC and biliary tract cancers not curable by local therapies with combination treatment of tremelimumab, a CTLA-4-blocking antibody and durvalumab, a PD-L1-blocking antibody along with ablative therapies such as radiofrequency ablation (NCT02821754). The hypothesis of this study is the efficacy of immune checkpoint inhibitors can be enhanced by local ablative therapies of the liver lesion by releasing tumor antigens systemically and promoting a systemic antitumor immune response. The combination of checkpoint inhibitors has been previously studied in other malignancies. For example, in melanoma combining nivolumab, a PD-1-blocking antibody and ipilimumab, a CTLA-4-blocking antibody, produced a greater objective response rate and progression-free survival as compared with single-agent therapy. However, at the cost of better response rates, combination therapy induced more adverse events [6]. Here, we report a case of a 61-year-old male with advanced HCC who developed a life-threatening shellfish allergy after completing three cycles of combination tremelimumab and durvalumab therapy.

Future Medicine 


\section{Case presentation}

A 61-year-old male with history of hepatitis C, Child Pugh B liver cirrhosis and hepatocellular carcinoma had progressive disease while on sorafenib therapy and was subsequently enrolled in our clinical trial with combination treatment of tremelimumab and durvalumab along with ablative therapies. The patient reported no known allergies prior to the initiation of immunotherapy. The patient completed three cycles of checkpoint inhibitor therapy with a partial tumor response and no drug- or immune-related adverse events. Before the initiation of the fourth cycle of therapy, the patient consumed a piece of shrimp and developed a severe anaphylactic reaction resulting in cardiac arrest. The patient had no history of adverse reactions to shellfish having eaten shrimp approximately 2 days prior with no adverse events. Cardiopulmonary resuscitation was performed with administration of diphenhydramine and epinephrine resulting in return of spontaneous circulation. He recovered from anaphylactic shock at a local community hospital and was subsequently discharged home. The patient consulted with an allergy specialist who confirmed the patient to have a new allergy to shellfish and shrimp. The patients continued combination treatment with tremelimumab and durvalumab with no additional adverse events reported.

\section{Discussion}

One of the first successful treatments using an immune-based therapy was in 1984 by Steven Rosenberg who studied IL-2 for the treatment of metastatic melanoma [7]. Since that time, a variety of immune-based therapies have been developed and include cytokine-based therapy, cell-based therapy, vaccine-based therapy and antibody-based therapy. Unlike traditional chemotherapy, immunotherapy acts indirectly through modulating the patient's immune system to promote tumor recognition and an antitumor response [4]. As the immune system is designed with checks and balances to prevent widespread immune activation and subsequent autoimmune disease, immunotherapy may produce a unique adverse event profile with the potential to cause irAEs by unbalancing the immune system where one may develop autoimmune manifestations [5]. There are a variety of irAEs that may occur when on checkpoint inhibitors including but not limited to pneumonitis, hypophysitis, endocrine dysfunction, skin reactions such as pruritus and rash, as well as hepatitis, transaminitis, diarrhea and colitis [5]. However, upon review of literature, little, if anything, is reported about development of a severe allergic reaction after immune checkpoint blockade.

Increasing numbers of clinical trials using checkpoint inhibitors gained new data about irAEs [5,8-10]. The quality and completeness of irAE reporting was found to be suboptimal and heterogeneous but improving over time, which may reflect growing recognition and familiarity with unique features of irAEs [11]. Combination of checkpoint inhibitors has been previously studied in melanoma combining nivolumab, a PD-1-blocking antibody, and ipilimumab, a CTLA-4-blocking antibody. It was found that combination therapy with immune checkpoint inhibitors produced a greater objective-response rate and progression-free survival as compared with single-agent therapy. However, combination therapy also produced greater drug-related adverse events with increase in grade 3 or 4 toxicities in $54 \%$ of patients with combination therapy compared with $24 \%$ of patients with monotherapy [6].

Immuno-oncology agents have been described to produce hypersensitivity reactions in previous studies. IL-2 has been reported to promote hypersensitivity reactions in patients receiving iodine containing radiographic contrast media at a higher incident than the general population $[12,13]$. Preclinical studies highlighted fatal hypersensitivity reactions in mice with $4 \mathrm{~T} 1$ tumors exacerbated by blockage of PD-1/PD-L1 [14]. Adverse reactions to shellfish can be produced by immunological and nonimmunological reactions. Nonimmunologic reactions tend to be triggered by contaminants such as parasites, bacteria, viruses or other toxins while immunologic reactions tend to be related to the allergen tropomyosin, arginine kinase or myosin light chain and may or may not be mediated by IgE [15]. The exact mechanism by which immune checkpoint inhibitors may cause the development of a new allergy is not fully understood and may be due to the imbalance of the immune system with the activation of $T$ cells and subsequent release of cytokines. Here, we report a timewise correlation of dual immune checkpoint blockade with the development of a new and severe shellfish allergy leading to anaphylactic shock. There are no parameters to produce accurately a chain of causation linking this event to dual checkpoint blockade. However, the patient's history of no reported allergy, the severity of the allergic reaction and the temporal connection encouraged us to report this case to sensitize oncologists for this possible type of IRAE.

\section{Future perspective}

As progress in the development of new systemic therapies for $\mathrm{HCC}$ has been slow, positive results from clinical trials involving immunotherapy have been promising. Recently, the US FDA approved nivolumab for the treatment of 
HCC based on the results of the Phase I/II CheckMate-040 trial [16]. These results show promise in the development and possible combination of immunotherapies for the treatment of HCC.

\section{Executive summary}

- The use of immune checkpoint inhibitors appears to be a viable option and has recently been approved by the US FDA for the treatment of hepatocellular carcinoma in patients who have progressed with sorafenib.

- Unlike traditional chemotherapy which has direct cytotoxic effects on cancer cells, immunotherapy acts through modulating the patient's immune system to promote tumor recognition and an antitumor response. As a result, immune checkpoint inhibitors may produce unique immune-related adverse events.

- Clinicians must be aware of the potential of immune-related adverse events that occur in patients on immunotherapies.

\section{Financial \& competing interests disclosure}

The authors are supported by the Intramural Research Program of the NIH, NCI. TF Greten is an employee of the US Federal Government. The authors have no other relevant affiliations or financial involvement with any organization or entity with a financial interest in or financial conflict with the subject matter or materials discussed in the manuscript apart from those disclosed.

No writing assistance was utilized in the production of this manuscript.

\section{Ethical conduct of research}

The authors state that they have obtained appropriate institutional review board approval or have followed the principles outlined in the Declaration of Helsinki for all human or animal experimental investigations. In addition, for investigations involving human subjects, informed consent has been obtained from the participants involved.

\section{References}

1. Ryerson AB, Eheman CR, Altekruse SF et al. Annual report to the Nation on the status of cancer, 1975-2012, featuring the increasing incidence of liver cancer. Cancer 122(9), 1312-1337 (2016).

2. Bruix J, Qin S, Merle P et al. Regorafenib for patients with hepatocellular carcinoma who progressed on sorafenib treatment (RESORCE): a randomised, double-blind, placebo-controlled, Phase III trial. Lancet 389(10064), 56-66 (2017).

3. Llovet JM, Ricci S, Mazzaferro V et al. Sorafenib in advanced hepatocellular carcinoma. N. Engl. J. Med. 359(4), 378-390 (2008).

4. Prieto J, Melero I, Sangro B. Immunological landscape and immunotherapy of hepatocellular carcinoma. Nat. Rev. Gastroenterol. Hepatol. 12(12), 681-700 (2015).

5. Michot JM, Bigenwald C, Champiat S et al. Immune-related adverse events with immune checkpoint blockade: a comprehensive review. Eur. J. Cancer 54, 139-148 (2016).

6. Postow MA, Chesney J, Pavlick AC et al. Nivolumab and ipilimumab versus ipilimumab in untreated melanoma. N. Engl. J. Med. 372(21), 2006-2017 (2015).

7. Rosenberg SA. IL-2: the first effective immunotherapy for human cancer. J. Immunol. 192(12), 5451-5458 (2014).

8. Abdel-Wahab N, Shah M, Suarez-Almazor ME. Adverse events associated with immune checkpoint blockade in patients with cancer: a systematic review of case reports. PloS ONE 11(7), e0160221 (2016).

9. Maughan BL, Bailey E, Gill DM, Agarwal N. Incidence of immune-related adverse events with program death receptor-1- and program death receptor-1 ligand-directed therapies in genitourinary cancers. Front. Oncol. 7, 56 (2017).

10. Byun DJ, Wolchok JD, Rosenberg LM, Girotra M. Cancer immunotherapy - immune checkpoint blockade and associated endocrinopathies. Nat. Rev. Endocrinol. 13(4), 195-207 (2017).

11. Chen TW, Razak AR, Bedard PL, Siu LL, Hansen AR. A systematic review of immune-related adverse event reporting in clinical trials of immune checkpoint inhibitors. Ann. Oncol. 26(9), 1824-1829 (2015).

12. Choyke PL, Miller DL, Lotze MT, Whiteis JM, Ebbitt B, Rosenberg SA. Delayed reactions to contrast media after interleukin-2 immunotherapy. Radiology 183(1), 111-114 (1992).

13. Zukiwski AA, David CL, Coan J, Wallace S, Gutterman JU, Mavligit GM. Increased incidence of hypersensitivity to iodine-containing radiographic contrast media after interleukin-2 administration. Cancer 65(7), 1521-1524 (1990).

14. Mall C, Sckisel GD, Proia DA et al. Repeated PD-1/PD-L1 monoclonal antibody administration induces fatal xenogeneic hypersensitivity reactions in a murine model of breast cancer. Oncoimmunology 5(2), e1075114 (2016).

15. Lopata AL, O’Hehir RE, Lehrer SB. Shellfish allergy. Clin. Exp. Allergy 40(6), 850-858 (2010). 
16. El-Khoueiry AB, Sangro B, Yau T et al. Nivolumab in patients with advanced hepatocellular carcinoma (CheckMate 040): an open-label, non-comparative, Phase I/II dose escalation and expansion trial. Lancet 389(10088), 2492-2502 (2017). 